\title{
The Need and Scope for Stem Cell Based Therapy in Hirschsprung Disease
}

\section{Thambidorai $\mathrm{CR}^{*}$}

Paediatric surgery unit, Department of Surgery, University Malaya Medical Centre, University Malaya, 50603, Kuala Lumpur, Malaysia

*Corresponding author: Thambidorai CR, Professor and Senior consultant surgeon, Paediatric surgery unit, Department of Surgery, University Malaya, 50603, Kuala Lumpur, Malaysia, Tel: 6-03-79492441; Email: thambidorai@gmail.com

\section{Abstract}

The role of enteric nervous system in gastrointestinal motility disorders in children has been better understood in recent years. One of the well-researched among these disorders is Hirschsprung disease (HSCR), also known as congenital intestinal aganglionosis. Even with current advances in the surgical management of HSCR significant postoperative morbidity such as defecation problems and enterocolitis occur despite correctly performed surgery. The need for adopting alternate forms of therapy in HSCR disease is well realized and stem cell therapy may provide this path in future.

The understanding of embryology of neural crest stem cells and enteric nervous system progenitor cells and their migration to locations in the gut provided the early clues for stem cell therapy in HSCR. Identification of these cells in postnatal tissues at various anatomical locations including the intestines of infants, children and even patients with HSCR has provided the impetus for more intense research in this area. The autologous source of neural crest stem cells from the gut of HSCR patients is a major development in not being immunogenic. Despite these advances, several issues still remain to be solved before adoption of stem cell therapy for HSCR in humans.

This mini-review highlights the current status and future directions of the role of stem cell therapy in therapy of HSCR.

Keywords: Hirschsprung Disease; Neural Crest Stem Cell; Enteric Nervous System; Stem Cell Therapy; Enteric Nerves Forming Stem Cells

Abbreviations: HSCR: Hirschsprung Disease; ENS: Enteric Nervous System; NCSC: Neural Crest Stem Cells; Nlbs: Neurosphere-Like Bodies; ENSC: Enteric Nerve
Forming Stem Cells; GDNF: Glial Cell Line Derived Neurotrophic Factor. 


\section{Journal of Embryology \& Stem Cell Research}

\section{Introduction}

In paediatric surgical practice, patients with congenital or acquired gastrointestinal motility disorders constitute a significant proportion. The etiology, presentation, severity, management issues, short and long term outcomes of therapy and health care costs of these disorders fall into a very wide spectrum [1,2] Hirschsprung disease (HSCR), internal sphincter achalasia, and pseudo obstructions are a few examples of such disorders seen in children.

Over the past few decades, the role of enteric nervous system (ENS) in these motility disorders has been better understood. One of the well-researched of these disorders is HSCR which is also the most common of the ENS abnormalities seen in children [1-3]. The affected segment of bowel in HSCR is aganglionic and tonically contracted. The aganglionic segment extends from the anus proximally to rectosigmoid junction in about $70 \%$ of HSCR patients, to more proximal levels of colon in $20 \%$ and the whole of colon in less than 10\% [3-5].

Despite the modern improvements in diagnosis and therapy of HSCR, post-surgical outcome still shows significant morbidity [1,2]. The morbidity includes persisting constipation, fecal incontinence, HSCR associated enterocolitis and rarely need for permanent stoma. The causes for these are many and may reflect dysfunction of the residual ganglionic segment, abnormal anal sphincter function, retention of part of aganglionic distal bowel and sequelae of removal of part or whole of the rectum. Postoperative enterocolitis has been documented in the range of $4.6 \%$ to $54 \%$ with recurrent enterocolitis in about $5 \%[3,4]$. Enterocolitis carries a risk of mortality even now $[5,6]$. The need for additional modes of therapy in the armamentarium of surgeons managing HSCR is thus justified and stem cell therapy has provided hope in this direction $[3,5,6]$.

\section{ENS development and Basis for Stem Cell Use in HSCR}

The neural crest stem cells (NCSC) which migrate from the region of branchial arches to the bowel wall form the principal source of ENS cells [1,6,7]. These run along the vagal nerves to colonize the distal gut and travel the longest compared to any other set of cells during embryogenesis. In the pelvic region, the ENS is supplemented from sacral part of neural crest [1]. The neural crest-cells derived from sacral region reach the colon after the vagal neural crest cells but cannot by themselves avoid the occurrence of HSCR in the absence of vagal neural crest cells. A small population of vagal neural crest cells are sufficient to form ENS in the colon [1].

NCSC persist in the postnatal gut in animals and humans. Enteric nervous system progenitor cells derived from NCSC have been obtained from the bowel of embryonic, postnatal and adult animals (e g Mice and rat) and also the human gut in immediate postnatal period, in infants, in HSCR patients and normal adults [7,8]. ENS forming embryonic stem cells derived from various sources when grown in culture form neurospheres or neurosphere-like bodies (NLBs). The NLBs when transplanted into aganglionic embryonic mouse gut, differentiated into enteric nerve phenotypes at appropriate locations. The transplanted cells were capable of giving action potentials and receiving fast excitatory postsynaptic potentials $[1,9]$.

Lately, full thickness and submucosal biopsies (of size 1 $\mathrm{cm}$ or less) from infants have been expanded in culture to form NLBS capable of migration and differentiation into neurons similar to those obtained from animal sources $[7,8,10,11]$. The big advantage of using ganglionated bowel (from HSCR patients) as stem cell source is the avoidance of immune rejections in the recipient site [1114]. Extra mucosal laparoscopic (minimally invasive) biopsies would make it easy to obtain sufficient enteric nerve forming stem cells (ENSC) even in neonates with HSCR.

Aganglionic bowel also has been found to be source for ENSC ${ }^{11}$. Using cell sorting technique p75-positive NCSC derived from thickened nerve trunks in aganglionic region of HSCR patients have been shown to form neurons in culture. This raises the possibility of their usage in autologous transplantation and inducing differentiation of these endogenous cells in situ as a therapy for aganglionosis [6,11]. The ganglionic and aganglionic bowels of short segment and long segment HSCR are better sources of ENSC when compared to bowel of total colonic and total intestinal aganglionosis [2].

\section{Future directions of research}

The above developments have significantly improved the possibility of using stem cells in HSCR therapy [1,2]. Future directions of research need to focus on deciding the appropriate source of stem cells, designing suitable models of aganglionic bowel to study effects of stem cell transplantation and correct ways of assessing cell migration, differentiation, connectivity, and function in 


\section{Journal of Embryology \& Stem Cell Research}

the transplanted bowel. Studies should aim to explore the results of stem cell transplantation in both embryonic and postnatal conditions $[13,14]$.

For clinical use of stem cell therapy in HSCR many practical challenges remain to be overcome. The ENSC derived from vagal distribution of NCSC have limited potential to generate ENS cells in vitro. Bone marrowderived mesenchymal stem cells have also shown promise as a source of autologous stem cells for ENSC generation but require manipulation of the soluble factors in their growth mediu [15]. Cell sorting in culture using markers such as Ret and p75 may provide a better source of ENS stem cells. Use of gut derived soluble factors appears to drive CNS-derived neuronal progenitor cells towards enteric phenotype development [16].

Gene-environment interactions affect ENS development and play a role in pathogenesis of HSCR [17]. Current research has shown that the migratory capacity of transplanted cells along the length of the gut has been limited to few millimeters whereas the length of aganglionic segment in the most common type of HSCR is at least $10 \mathrm{~cm}$. Microenvironment in the recipient gut and that of migrating cells such as glial cell line derived neurotrophic factor (GDNF) may play a part in promoting the migratory potential of transplanted cells. GDNF exposure in culture produced very significant increase in ENS cell number and neurosphere volume. Such GDNF treated cells when transplanted into the colon of postnatal mice in vivo, migrated twice the distance compared with controls [18]. Identification of such neurotrophic factors and treating or loading the stem cells with these factors is another area that needs further evaluation $[19,20,21]$. In HSCR patients with GDNF gene mutation, decreased expression of GDNF is expected in the aganglionic bowel. But surprisingly, decreased GDNF in the bowel has been shown even in the absence of GDNF mutation [21].

Mutations in at least 16 genes have been implicated in the development of HSCR of which RET has been the major mutation studied [22]. Peyer's patches of the gut have been found to be reduced in Ret 51/51 mutant mice. The role of RET signaling in gut immunity may explain the susceptibility to enterocolitis even after conventional corrective surgery. Modification of the recipient environment may be an area for research to avoid post therapy enterocolitis [6,19]. Apart from genetic factors, dietary nutrients and drugs influence the structure and function of ENS before and after birth. These have a possible role in the pathogenesis of HSCR (and irritable bowel syndrome) and can potentially be used in prevention of HSCR [17,23].
Defining the effective routeto deliver the stem cells to the aganglionic recipient gut and the number of injections required are other considerations in stem cell therapy. The stem cells can be injected throughthe serosa of the gut via laparotomy or laparoscopy. Intraperitoneal and intravenous injections are other possible choices [1]. The duration of viability of cells after successful implantation, the potential for neoplastic changes and the group of HSCR patients most likely to benefit from stem cell therapy are further aspects that require more definite answers.

\section{Conclusion}

The ENS therapy for HSCR would have a definite role to play in management of HSCR in future though many hurdles remain. Obtaining autologous stem cells has the advantages of good supply and avoidance of immunological reactions. Use of minimally invasive procedures helps in obtaining the stem cells easily and also delivering them back into gut. Rigorously monitored clinical trials with formulation of good practice guidelines would pave the way for optimal use of stem cell therapy in HSCR patients reinforcing what Louis Pasteur said in 1854 that "Chance favours the prepared mind".

\section{References}

1. Hotta R, Natarajan D, Thapar N (2009) Potential of cell therapy to treat pediatric motility disorders. Semin Pediatr Surg 18: 263-273.

2. Bethell G, Wilkinson D, Fawkner-Corbett D, Mesa A, Shukla R, et al. (2016) Enteric nervous system stem cells associated with thickened extrinsic fibers in short segment aganglionic Hirschsprung's disease gut are absent in the total colonic and intestinal variants of disease. J Pediatr Surg 51(10): 1581-1584.

3. Chung PHY, Wong KK, Leung JL, Tam PKH, Chung KL, et al. (2015) Clinical and manometric evaluations of anorectal function in patients after transanalendorectal pull-through operation for Hirschsprung's disease: A multicentre study. Surgical Practice 19(3): 113-119.

4. Giuliani S, Betalli P, Narciso A, Grandi F, Midrio P, et al. (2011) Outcome comparison among Laparoscopic Duhamel, laparotomic Duhamel, and transanalendorectal pull-through: A single-center, 18Year Experience. J Laparoendo Adv Surg Tech 21(9): 859-863. 


\section{Journal of Embryology \& Stem Cell Research}

5. Scholfield DW, Ram AD (2016) Laparoscopic Duhamel Procedure for Hirschsprung's Disease: Systematic Review and Meta-analysis. J Laparoendo Adv Surg Tech 26(1): 53-61.

6. Wilkinson, DJ, Edgar DH, Kenny SE (2012) Future therapies for Hirschsprung's disease. Semin Pediatr Surg 21(4): 364-370.

7. Metzger M, Caldwell C, Barlow AJ, Burns AJ, Thapar N (2009) Enteric nervous System stem cells derived from human gut mucosa for the treatment of aganglionic gut disorders. Gastroenterology, 136 (7): 2214-2225.

8. Metzger M, Bareiss PM, Danker T, Wagner S, Hennenlotter J, et al. (2009) Expansion and Differentiation of Neural Progenitors Derived From the Human Adult Enteric Nervous System. Gastroenterology 137(6): 2063-2073.

9. BurnsAJ, Roberts RR, Bornstein JC, Young HM (2009) Development of the enteric nervous system and its role in intestinal motility during fetal and early postnatal stages. Semin Pediatr Surg 18(4): 196-205.

10. Almond S, Lindley RM, Kenny SE, Connell MG, Edgar DH (2007) Characterisation and transplantation of enteric nervous system progenitor cell. Gut 56(4): 489-496.

11. Wilkinson DJ., Bethell GS, Shukla R, Kenny SE, Edgar DH (2015) Isolation of enteric nervous system progenitor cells from the aganglionic gut of patients with Hirschsprung's disease. PLoS ONE 10(5).

12. El-Nachef W, Grikscheit (2014) Enteric nervous system cell replacement therapy for Hirschsprung disease: beyond tissue-engineered intestine. Eur J PediatrSurg 24(3): 214-218.

13. Heanue TA, Pachnis V (2007) Enteric nervous system development and Hirschsprung's disease: Advances in genetic and stem cell studies. Nat Rev Neurosci 8(6): 466-479.

14. Burns AJ, Goldstein AM, Newgreen DF, Stamp L, Schäfer KH, et al. (2016) White paper on guidelines concerning enteric nervous system stem cell therapy for enteric neuropathies. Dev Biology 417(2): 229251.

15. Lin R, Ding Z, Ma H, Shi H, Gao Y, et al. (2015) In-vitro conditioned bone marrow derived mesenchymal stem cells promote de-novo functional enteric nerve regeneration, but not through directtransdifferentiation. Stem cells 33(12): 3545-3557.

16. Kulkarni S, Zou B, Hanson J, Micci MA, Tiwari G, et al. (2011) Gut-derived factors promote neurogenesis of CNS-neural stem cells and nudge their differentiation to an enteric-like neuronal phenotype. Am J PhysiolGastrointest Liver Physiol 301(4): G644-655.

17. Heuckeroth RO, Schäfer KH (2016) Geneenvironment interactions and the enteric nervous system: neural plasticity and Hirschsprung disease prevention. Dev Biol 417(2): 188-197.

18. McKeown SJ, Mohsenipour M, Bergner AJ, Young HM, Stamp LA (2017) Exposure to GDNF enhances the ability of enteric neural progenitors to generate an enteric nervous system. Stem Cell Reports 8 (2): 476488.

19. Bitar KN, Raghavan S, Zakhem E. (2014) Tissue engineering in the gut: Developments in neuromusculature. Gastroenterology 146(7): 16141624.

20. Tam, PKH, Chung PHY, St Peter SD, Gayer CP, Ford HR, et al. (2017) Advances in paediatric gastroenterology. The Lancet 390(10099): 1072-1082.

21. Tam PKH (2016) Hirschsprung's disease: A bridge for science and surgery. J Pediatr Surg 51(1): 18-22.

22. Nor Azian AM, Then SM, MohdRidhwan AR, Thambidorai CR, Noraima Othman, et al. (2017) Preliminary study shows novel variant detected in the screening of RET gene in Malaysian patients with Hirschsprung's disease; Asia-Pacific J Mol. Medicine 7(1): 1-6.

23. Burns AJ, Hofstra RMW (2016) The enteric nervous system: From embryology to therapy. DevlBiol 417(2): 127-128. 